\title{
Study on Identification of Face in Versatile Circumstances through Genetic and Ant Colony Optimization Algorithms
}

\author{
S. Venkatesan ${ }^{1}$ and S. Srinivasa Rao Madane ${ }^{2^{*}}$ \\ 'Department of Computer Science and Engineering, Dayananda Sagar College of Engineering, Bangalore- 560078, \\ Karnataka, India;Venkatesanselvam-cs@dayanandasagar.edu, selvamvenkatesan@gmail.com \\ 2 Department of Computer Science and Engineering, Adhiparasakthi Engineering College, \\ Kalavai-603319, Tamil Nadu, India; raomadane@gmail.com
}

\begin{abstract}
Background/Objectives: Image pre-processing is done for enhancing the features of the input image for efficient image recognition. Image enhancement is done in a series of steps by employing different filtering techniques. Those steps are discussed as follows: Histogram equalization and Normalization. Methods/Statistical Analysis: Research on Identification of Face in a Multifaceted Condition by clustering Enhanced Genetic and Ant Colony Optimization Algorithms. Results: The edge detection of the clipped face image. There are a number of different face recognizing and identification method which varies on different grounds and are employed for different scenarios. Those methods are employed in the system for which FIS is developed and their results are compared. Conclusion/Application: This Face Identification System by clustering Genetic and Ant Colony Optimization algorithm shows the maximum efficiency of $96 \%$. This ACOGA competence can be greater than before by using better face scanner, best technique of scaling and well organized technique of edge detection and feature extraction of the face image. The problems faced while working on this research work are the difficulties in detecting faces of overlapping face images and detecting different face poses.
\end{abstract}

Keywords: Ant Colony Optimization, Face Identification, Face Image Acquisition, Genetic Algorithm, Image Pre-Processing

\section{Introduction}

The goal of computer vision is the ability of computers is to achieve visual recognition ability comparable to that of human. Among many recognition subjects, face recognition has drawn considerable interest and attention from many researchers for the last twenty years because of its possible applications, such as in the areas of surveillance, secure trading terminals, Closed Circuit Television (CCTV) control, user authentication, Human Computer Interface (HCI), Humanoid robot. In this paper we propose a novel face identification system that employs two different soft computing techniques for efficient face recognition and identification.

\section{Face Identification System}

The proposed face identification system can be divided in to two major entities. They are the image pre-processing system followed by face identification system ${ }^{1}$. The image pre-processing system is used for image enhancement clipping and filtering the given image for easy and efficient feature extraction in the later stage of face identification. Further the edges of the image are detected by using $\mathrm{ACO}$ algorithm. Then using GA the detected edges are compared with the available database of images and the best fitting image is identified. A special advantage of the proposed technique is that there is no extra learning process included here, only by saving the

*Author for correspondence 


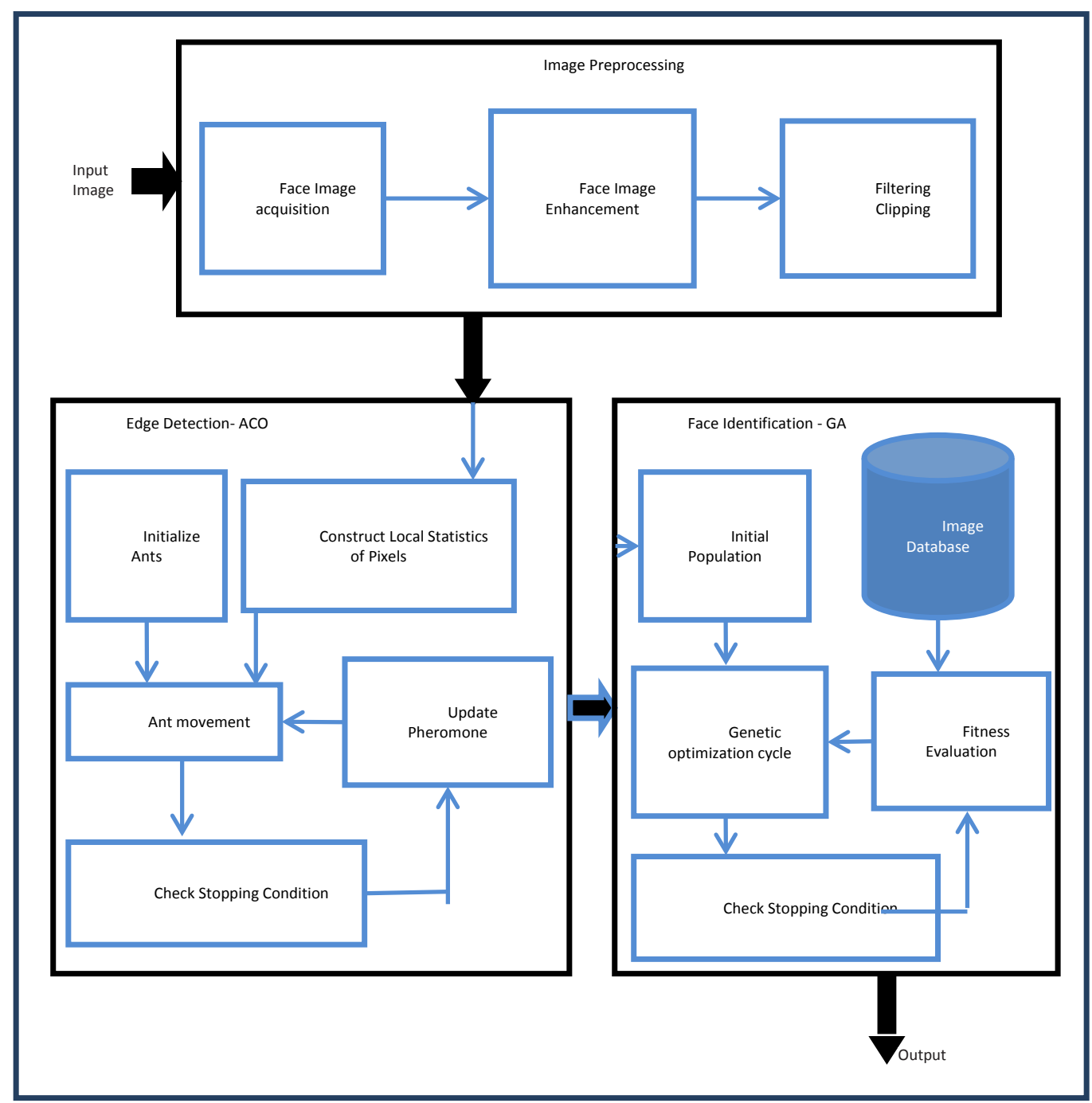

Figure 1. Basic work flow of FIS.

face information of the person and appending the person's name in the learned database completes the recognizing process. The image shown in Figure 1 shows the basic work flow of the Face Identification System (FIS) discussed in this work.

\section{Face Image Acquisition}

Before we start to do face identification or even image enhancement, we have to acquire the raw data of image for further processing. Any image data is an array of pixel values which can be represented as a matrix, where the rows and column are the image dimension itself. The number of bits used to represent the pixel values of component images determines the bit depth of an RGB image. An RGB color image is an $\mathrm{M} \times \mathrm{N} \times 3$ array of triplet data corresponding to the red, green and blue components of an RGB image. $\mathrm{M}$ and $\mathrm{N}$ are the number of pixels corresponding to the length and width of the image. The range of values is $[0,255]$ or $[0,65535]$ for RGB images of class unit 8 or unit 16 respectively. The number of possible colors in an RGB image is $(2 b)^{3}$, where $b$ is the number of bits in each component image. For 8-bit case, the number is $16,777,216$ colors. The RGB is converted into gray scale images for further image processing. These numerical data are used for image enhancement in the former and face detection and identification in the later stage. The image in Figure 2 shows the sample image used in this paper. 
The sample RGB image is converted to gray scale image for better image processing. The edges of the image can be detected efficiently in gray scale mode than in RGB mode. Hence the image is converted to gray scale mode before further enhancement techniques are employed. Figure 3 shows the gray scale image of the RGB image shown in Figure 2. Figure 4 shows the sample numerical data of the image shown in Figure 2.

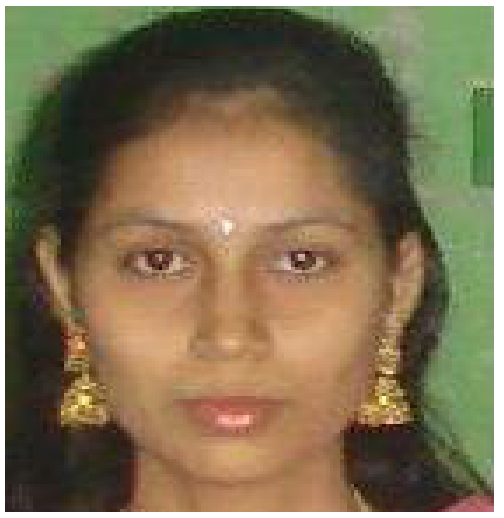

Figure 2. Sample RGB image.

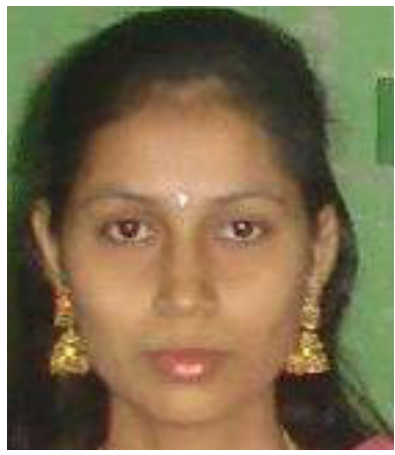

Figure 3. Sample Gray Scale image.

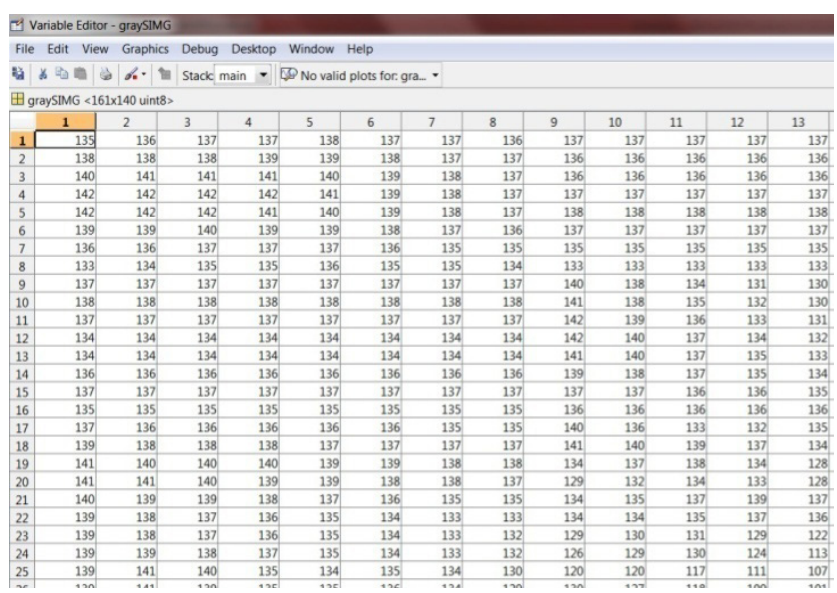

Figure 4. Sample numerical data of Gray Scale image in 'Figure 2'.

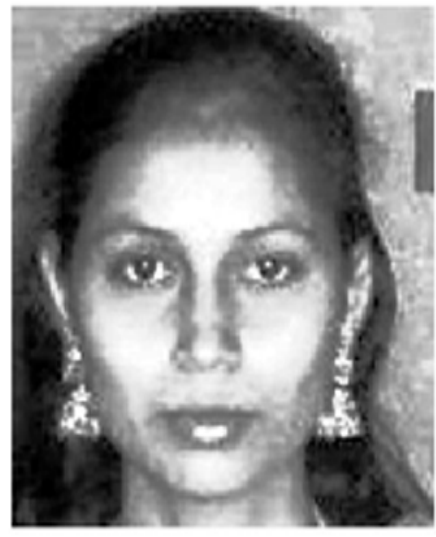

Figure 5. Histogram equalized image.

\section{Image Pre-Processing}

Image pre-processing is done for enhancing the features of the input image for efficient image recognition. Image enhancement is done in a series of steps by employing different filtering techniques. Those steps are discussed as follows.

\section{Histogram Equalization and Normalization}

After the image is converted to gray scale the image data is enhanced by equalizing the histogram of the image, histogram equalization is done to enhance the contrast of the image. After enhancing the contrast of the image, it is normalized so that the possibility of edge detection is improved.

The histogram equalized image is shown in Figure 4. It is clearly seen that the contrast of the image is enhanced when the histogram of the image is equalized. The histogram of the image is shown in Figure 5.

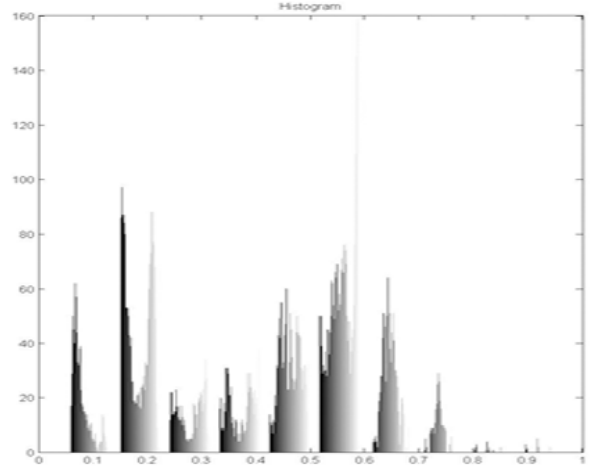

Figure 6. Histogram of Figure 4. 


\section{Filtering and Clipping}

The input image is prone to contain noise which should be removed. There are different types of noise those affect the image ${ }^{1}$. They are Gaussian, Salt and Pepper, Shot, uniform, film grain and Non-Isotropic noise which would damage the image data. To efficiently remove this noise prewitt filtering technique is used. The filtered image is shown in Figure 7.

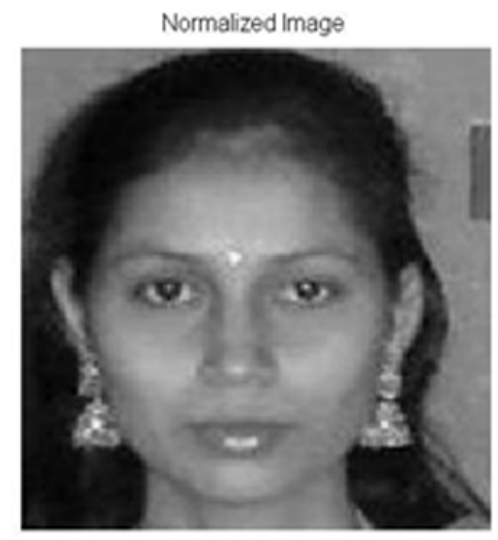

Figure 7. Normalized image after histogram equalization.

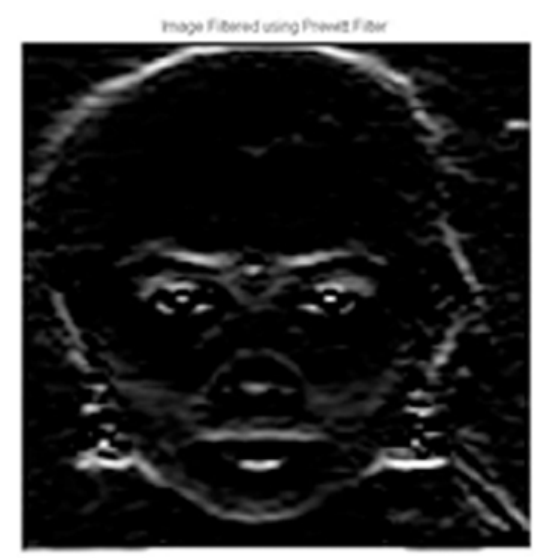

Figure 8. Image shown in Figure 6 Filtered using Prewitt filtering technique.

This is a non-linear digital filter often used to remove noise in an image. Noise reduction helps us to improve the results in the later stages of the face identification. Prewitt filter is used exclusively for noise removal, because it removes noise from the image without damaging the edges of the image. To improve both the image and the quality of filtering, the filtered image is dilated and eroded. Dilating and Eroding the image is called as morphological operations. The dilated and eroded image is shown in Figure 8.

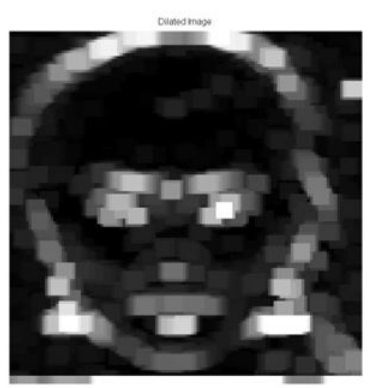

(a)

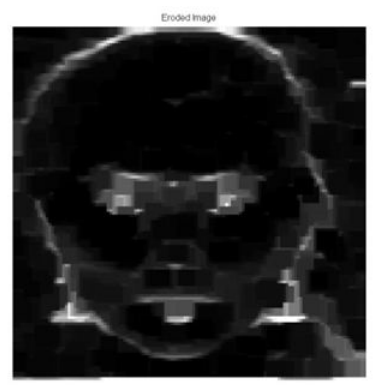

(b)
Figure 9. Morphological operations. (a) Dilated image. (b) Eroded image.

After the morphological operations, the image is clipped to obtain the necessary data that is required for further processing of the input image. The noise reduced image is shown in Figure 9.

With these three sections described above the image pre-processing is over and the image is ready to be identified. Thus the image is presented as input to the next stages of the FIS.

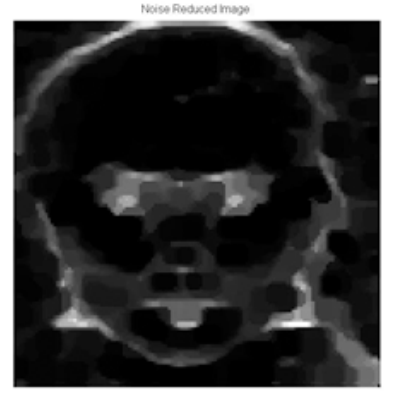

Figure 10. Noise reduced image.

\section{Face Identification System (FIS)}

Our vision system works by identifying the edges of the scenes we see. Our brain automatically identifies the edges and clusters the edges of objects together. Thus our brain lets us to identify different objects easily by identifying edges and clustering them automatically. Thus for implementing image recognition for an artificial intelligent system the edges of the image should be identified. To identify the edges, we have used Ant Colony Optimization (ACO). ACO is an evolutionary optimization technique which is based on the act of ants foraging for 
food. The identified edges are cross checked by Genetic Algorithm (GA) with a database of images, which identifies the availability of a particular image in the database. Thus the face is first detected and then identified from the database of available images.

\section{Edge Detection}

As discussed above, ACO is used for detecting edges of the image. In this algorithm the nodes represent features, with the edges between them denoting the choice of the next feature. The search for the optimal feature subset is then an ant traversal through the graph where a minimum number of nodes are visited that satisfies the traversal stopping criterion. The formula used in this technique is shown below in (1).

$$
p_{i j}^{k}(t)=\left\{\begin{array}{cc}
\frac{\left[\tau_{i j}(t)\right]^{\alpha} \cdot\left[\eta_{i j}\right]^{\beta}}{\sum_{l \in J_{i}^{k}}\left[\tau_{i l}(t)\right]^{\alpha} \cdot\left[\eta_{i l}\right]^{\beta}} & \text { if } j \in J_{i}^{k} \\
0 & \text { otherwise }
\end{array}\right.
$$

Where, $\mathrm{k}$ is the number of ants; $\eta_{\mathrm{ij}}$ is the heuristic desirability of choosing feature $j ; j$ is the set of neighbor nodes of node $i$ which has not yet been visited by the ant $k ; \tau$ is the amount of virtual pheromone on edge $(i, j)$. The pheromone is updated during each iteration by the formula shown in formula 2 and 3.

$$
\begin{gathered}
\tau_{i j}(t+1)=(1-\rho) \cdot \tau_{i j}(t)+\rho \cdot \Delta \tau_{i j}(t) \\
\Delta \tau_{i j}(t)=\sum_{k=1}^{n}\left(\gamma^{\prime}\left(S^{k}\right)\left|S^{k}\right|\right)
\end{gathered}
$$

Where $\rho$ is the pheromone evaporation coefficient. By employing this technique the edges of the image is identified. The use of ACO in our system is shown in Figure 10.

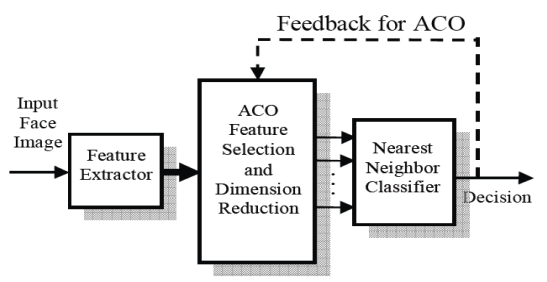

Figure 11. Use of ACO in FIS.

The edges are identified by employing different modes, thus we get a diversified types of edges of the same image, which improves the output of FIS. The edges detected by using different modes are shown in Figure 11.

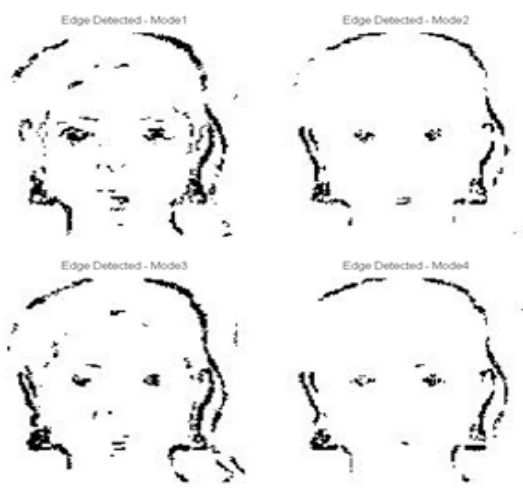

Figure 12. Edges detected by ACO by employing different modes.

\section{Face Detection}

In this work Genetic Algorithm is used for face identification. To get started with GA the initial population should be identified first. The initial population in our work is the image itself. The pixels of an image are the actual data of the image. The pixels of an image are encoded to form the chromosomes. 'N' number of such chromosomes constitutes a generation. Encoding the chromosome of a $10 \times 10$ pixel image is shown in the following images (Figure 12).

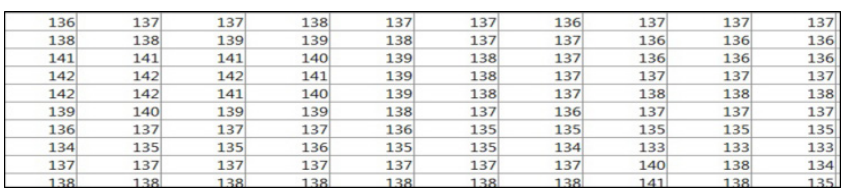

Figure 13. Pixels of a $10 \times 10$ image.

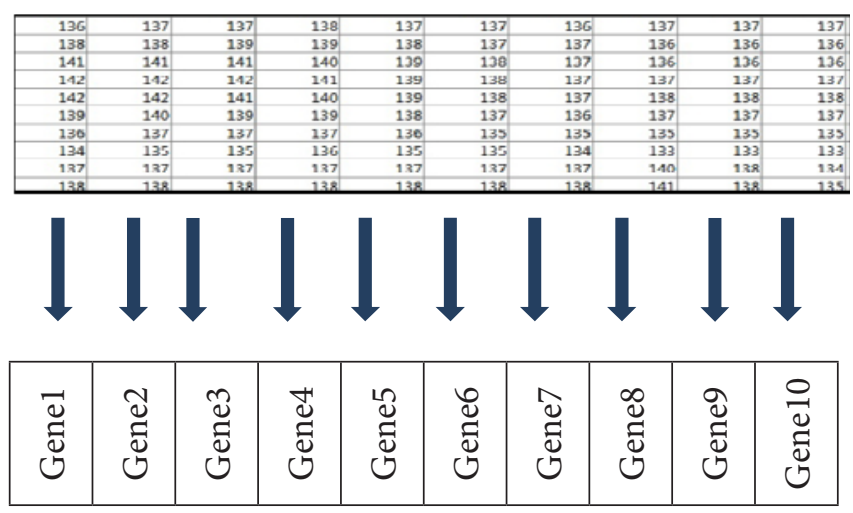

Figure 14. Representation of encoding pixels of an image to a chromosome. 
As shown in the representation, each gene is a vector of 10 pixel data. The chromosome consists of 10 genes, where each gene is a vector of 10 data. Thus all data of the image has been packed in to a chromosome. After generating the initial population, the GA routine is performed to find the best matching image from the database. To do this efficiently the pixel data of the edge detected image is encoded as the chromosome and compared with the database. The GA routine is as follows; selection, crossover, mutation and evaluation using fitness function.

The selection technique used in our work is roulette wheel selection technique (Figure 13). In this technique each chromosome is given a slice of the wheel. The area of the slice is based on the fitness value of the chromosome, obviously which indicates that the highly fit chromosomes occupy a wide area compared with the chromosomes with less fitness value.

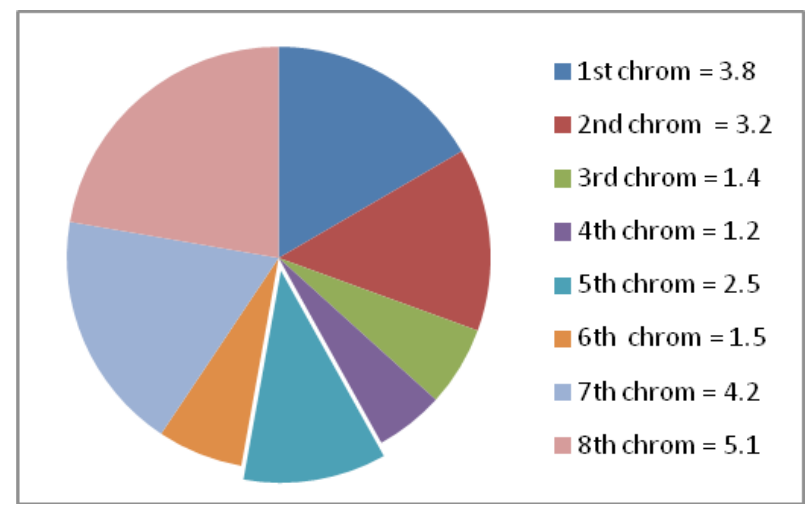

Figure 15. Roulette wheel, representing fitness of a chromosome.

To select the chromosome, a random number is generated between 0 and 100. The selected the chromosome is the one whose segment spans the random number. The selected chromosomes are subjected to crossover, so the features are exchanged which paves way to generate the best fit chromosomes and hence find the best matching chromosome. In this method we have employed multipoint crossover method. The multipoint crossover method is represented pictorially in the Figure 14.

The length of chromosome represented in the Figure 14 is 45 . The legends $\mathrm{P} 1, \mathrm{P} 2$ are the parent chromosomes 1 and 2 selected for crossover and C1, C2 are the children generated after the crossover process. It can be seen that the children have the properties of their parents mixed which may result as a good member with good fitness value or a bad member with low fitness value.

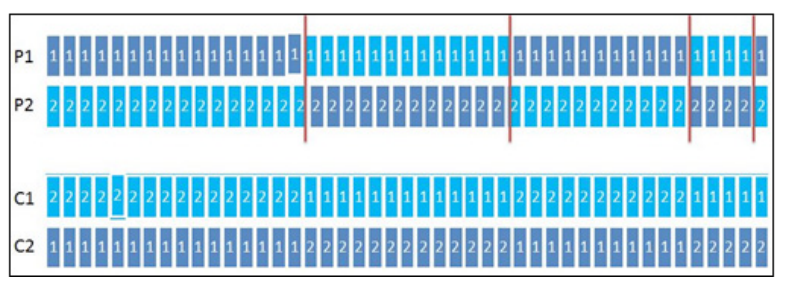

Figure 16. Representation multipoint crossover method.

The mutation is done to implement the evolutionary strategy in GA. To implement mutation a random bit is selected and the value is altered by replacing it with any random value that falls within the range of all possible values for a gene. In our work since we use grayscale images. The range is between 0 and 255. The mutation will either result in a good chromosome or a bad chromosome.

The Figure 15 shows the overall output of the processes of the genetic algorithm; the different parts of the images are described from left to right from the top as follows:

- Shows the fitness value of the best individual of each generation.

- Shows the values of the best genes of the current best individual.

- Shows the Best and Worst individual fitness value and also shows the mean fitness of each generation.

- Shows the best individuals selected for crossover and the number of children they produce.

- Shows the stopping criteria.

There are basically two stopping conditions which are based on the number of generations, for example we can stop the genetic process if the number of generations reaches 100 . We can also stop the genetic process if the individuals stall (i.e.) there is no more further development in the fitness of the individuals. After the stopping condition is achieved; decision is made whether the image has been identified or not.

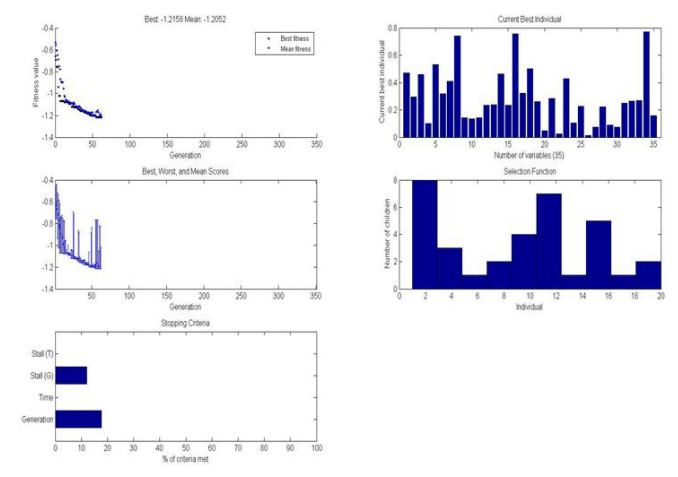

Figure 17. Shows the overall output of the Genetic algorithm. 


\section{Results and Discussion}

The image in Figure 16 shows the skin detection of the clipped face image. From the image shown it can be seen that the outline of the skin in the image is perfectly outlined. Based on the identified skin in the image the face is marked based on which the face identification is done perfectly.

The face marked image is shown in the Figure 17. Based on the detected face in the input image the final face identification process is perfected.

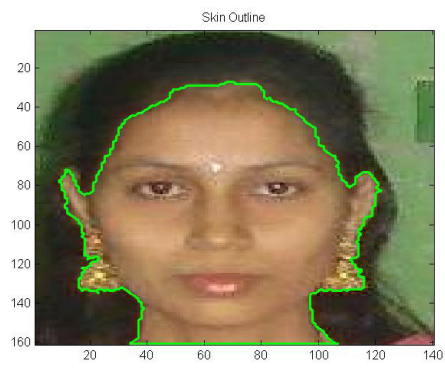

Figure 18. Shows the face skin outlined image.

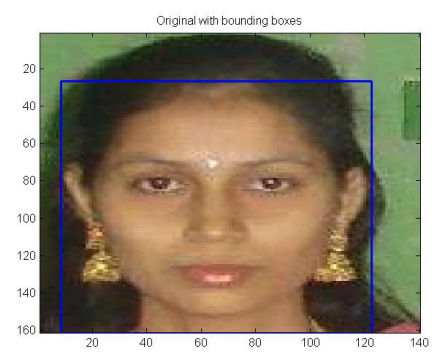

Figure 19. Shows the face detected and marked image.

The final face identification is done by genetic algorithm and hence the output is either the face is identified or not, both the cases are shown in the Figures 18 and 19. The success and failure of face identification means that either the input face image is available in the database or not available in the database (i.e.) either the face is authenticated or not authenticated.

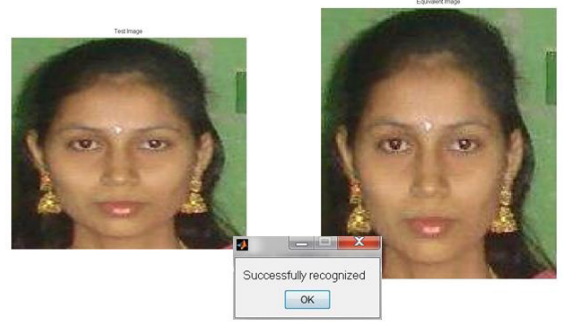

Figure 20. Shows the output of successful face identification.

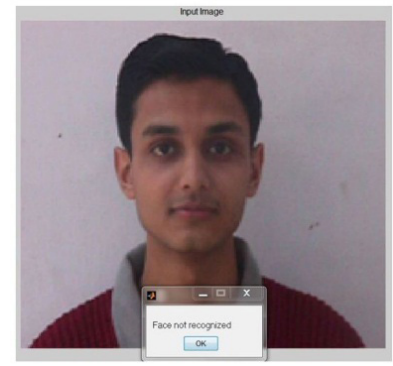

Figure 21. Shows the output of failed face identification.

From this system we have also generated a technique to detect multiple faces available in the input image. This is done by extracting the face features in the given image and hence count the number of faces based on clustering the face features. Though the algorithm is not perfect in detecting all the faces available in the image the output of the system is shown in the Figure 20.

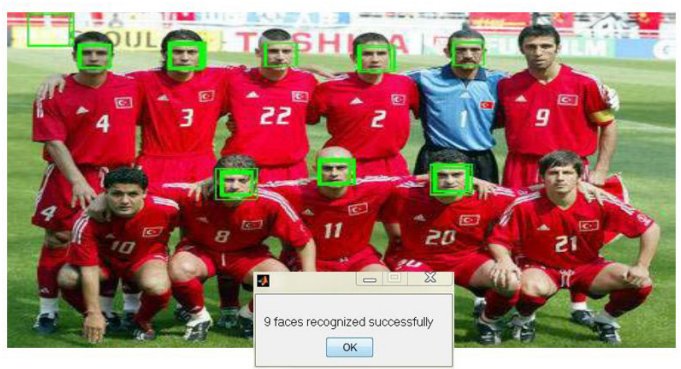

Figure 22. Multiple face detection.

A comparison chart is shown in the Table 1 shown below. Which compares the number of subjects used in the algorithm and the efficiency of the Face Identification System employed in this research work. The efficiency of ACOGA algorithm is shown in the Table 1 . The results of testing the algorithm with different number of subjects and the number of images left unrecognized are tabulated in the table. The same is represented graphically in the Figure 21.

Table 1. The efficiency of ACOGA

\begin{tabular}{|c|c|c|c|}
\hline Subjects & $\begin{array}{c}\text { Successfully } \\
\text { recognized Test } \\
\text { Image }\end{array}$ & $\begin{array}{c}\text { Unrecognized } \\
\text { Test Image }\end{array}$ & $\begin{array}{c}\text { Efficiency } \\
(\%)\end{array}$ \\
\hline 5 & 4 & 1 & 80 \\
\hline 10 & 8 & 2 & 80 \\
\hline 15 & 13 & 2 & 86.6 \\
\hline 20 & 18 & 2 & 90 \\
\hline 25 & 24 & 1 & 96 \\
\hline 30 & 28 & 2 & 93.3 \\
\hline
\end{tabular}




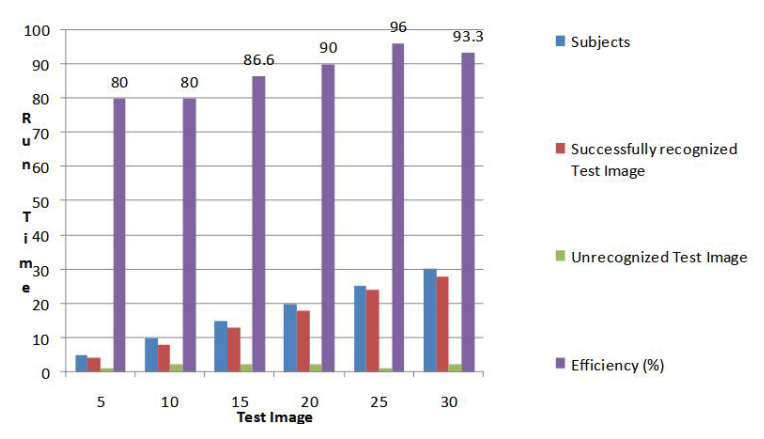

Figure 23. Graphical representation of efficiency of ACOGA.

There are a number of different face recognizing and identification method which varies on different grounds and are employed for different scenarios. Those methods are employed in the system for which FIS is developed and their results are compared in the Table 2 and the same is represented also.

It is clearly seen that our system tops the other methods with $96 \%$ efficiency. The graphical representation of the same is shown in the Figure 22.

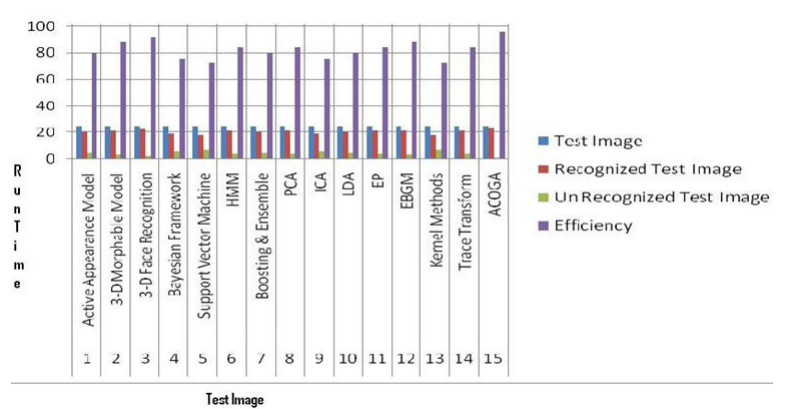

Figure 24. Graphical representation of efficiency of ACOGA when compared with other face identification algorithms.

\section{Conclusion}

In this research work, a prototype for Face Identification System using the Soft Computing techniques like Genetic algorithm and Ant colony optimization algorithm is discussed. The experimental analysis shows that the above methods are more robust and suitable for low

Table 2. The efficiency of ACOGA when compared with other algorithms

\begin{tabular}{|c|c|c|c|c|c|}
\hline S.No. & Method & Test Image & $\begin{array}{l}\text { Recognized Test } \\
\text { Image }\end{array}$ & $\begin{array}{l}\text { Un Recognized } \\
\text { Test Image }\end{array}$ & $\begin{array}{c}\text { Efficiency } \\
\%\end{array}$ \\
\hline 1 & $\begin{array}{c}\text { Active Appearance } \\
\text { Model }\end{array}$ & 25 & 20 & 5 & 80 \\
\hline 2 & $\begin{array}{l}\text { 3-D Morph able } \\
\text { Model }\end{array}$ & 25 & 22 & 3 & 88 \\
\hline 3 & $\begin{array}{c}\text { 3-D Face } \\
\text { Recognition }\end{array}$ & 25 & 23 & 2 & 92 \\
\hline 4 & $\begin{array}{c}\text { Bayesian } \\
\text { Framework }\end{array}$ & 25 & 19 & 6 & 76 \\
\hline 5 & $\begin{array}{l}\text { Support Vector } \\
\text { Machine }\end{array}$ & 25 & 18 & 7 & 72 \\
\hline 6 & HMM & 25 & 21 & 4 & 84 \\
\hline 7 & $\begin{array}{l}\text { Boosting and } \\
\text { Ensemble }\end{array}$ & 25 & 20 & 5 & 80 \\
\hline 8 & PCA & 25 & 21 & 4 & 84 \\
\hline 9 & ICA & 25 & 19 & 6 & 76 \\
\hline 10 & LDA & 25 & 20 & 5 & 80 \\
\hline 11 & $\mathrm{EP}$ & 25 & 21 & 4 & 84 \\
\hline 12 & EBGM & 25 & 22 & 3 & 88 \\
\hline 13 & Kernel Methods & 25 & 18 & 7 & 72 \\
\hline 14 & Trace Transform & 25 & 21 & 4 & 84 \\
\hline 15 & ACOGA & 25 & 24 & 01 & 96 \\
\hline
\end{tabular}


resolution, under shadow with different backgrounds and different facial expressions. This Face Identification System by clustering Genetic and Ant Colony Optimization algorithm shows the maximum efficiency of $96 \%$. This ACOGA competence can be greater than before by using better face scanner, best technique of scaling and well organized technique of edge detection and feature extraction of the face image. The problems faced while working on this research work are the difficulties in detecting faces of overlapping face images and detecting different face poses. In future the algorithm may be modified for different face angles and illumination variations and also to extend the algorithm to detect faces in video and live stream, hence we can detect faces in real time.

\section{References}

1. Persis DJ, Robert TP. Ant based multi-objective routing optimization in mobile ad-hoc network. Indian Journal of Science and Technology. 2015 May; 8(9):875-88.

2. Sasi SB, Sivanandam N. A survey on cryptography using optimization algorithms in WSNs. Journal of Science and Technology. 2015 Feb; 8(3):216-21.

3. Lakshmi Narayana KV, Kumar VN, Dhivya M, Raj RP. Application of ant colony optimization in tuning a PID controller to a conical tank. Indian Journal of Science and Technology. 2015 Jan; 8(S2):217-23.

4. Gandhe ST, Talele KT, Keskar AG. Face recognition using contour matching. International Journal of Computer Science. 2008 May 20; 35(2):1-8.

5. Venkatesan S, Madane SSR. Face detection by hybrid genetic and ant colony optimization algorithm. Int J Comput Appl. Foundation of Computer Science. 2010 Nov; 94(4):8-13.

6. Venkatesan S, Karnan M. Advanced classification using genetic algorithm and image segmnetation for improved face detection. Computer Research and Developement Second International Conference (ICCRD); 2010 May 7-10. p. 364-8.

7. Janaki Raam KV, Rajkumar K. A novel approach using parallel ant colony optimization algorithm for detecting routing path based on cluster head in wireless sensor network. Indian Journal of Science and Technology. 2015 Jul; 8(16):61986.

8. Anchugam CV, Thangadurai K. Detection of black hole attack in mobile ad-hoc networks using ant colony optimization - simulation analysis. Indian Journal of Science and Technology. 2015 Jul; 8(13):58200.

9. Samal, Iyengar PA. Automatic recognition and analysis of human faces and facial Expressions: A survey. Pattern Recognition; 1992.

10. Du S, Ward R. Wavelet-based illumination normalization for face recognition. Proc of IEEE Conference on Image Processing. 2005; 2:954-7.

11. Liu WG, Miao J, Zhao D, Deng G, Li J. Illumination compensation and feedback of illumination feature in face detection. Proc IEEE Int Conferences on Info-tech and Info-net. 2001; 23:444-9.

12. Georghiades AS, Belhumeur PN, Kriegman DJ. From few to many: Generative models for recognition under variable pose and illumination. IEEE Int Conf on Automatic Face and Gesture Recognition; 2000. p. 277-84.

13. Venkatesan S, Madane SSR. Face recognition system with genetic algorithm and ANT colony optimization. International Journal of Innovation, Management and Technology. 2010 Dec; 1(5). ISSN: 2010-0248.

14. Venkatesan S, Karnan M. Edge and characteristic subset selection in images using ACO. IEEE 2nd International Conference on Computer Research and Development; 2010 May 7-10. p. 369-72.

15. Venkatesan S, Madane SSR. A narrative approach for human face detection using ant colony optimization and genetic algorithm review. Adv Eng Informat. 2011; 33:2130-3. ISSN: 2229-712X. 